\title{
Diseño centrado en el usuario: Usabilidad web del portal de difusión cultural de leyendas riobambeñas
}

\author{
User-centered design: Website usability of the cultural diffusion portal of \\ riobambeñas legends
}

Mayra Luzmila Pomaquero Yuquilema. ${ }^{1}$, Bertha Alejandra Paredes Calderón. ${ }^{2}$, Andrea Fernanda Hidrobo Nina. ${ }^{3} \&$ Fabián Alfonso Calderón Cruz. ${ }^{4}$

Recibido: 15-05-2019 / Revisado: 18-06-2019 /Aceptado: 09-07-2019/ Publicado: 28-07-2019

\begin{abstract}
.
DOI: https://doi.org/10.33262/cienciadigital.v3i3.2.1.787

The research presents the study of the DCU User-Centered Design process of the Riobambeñas legends web portal, its content refers to cultural dimensions, worked from design techniques and whose result is reflected in digital platforms. To evaluate the designs made, two user tests have been carried out based on the heuristic principles and applied to two groups of users between 18 and 26 years of age at the Polytechnic School of Chimborazo. The results are mainly related to the experience provided by the new design, which has been valued very positively by the students, and it is obtained as a result that the creation of web portals designed to meet the needs of the user and the application of a test prior to the Publication of the portals allows you to have attractive websites that meet the criteria of an effective website.
\end{abstract}

Keywords: Web design, user design, cultural diffusion, legends

Resumen.

La investigación presenta el estudio del proceso de Diseño Centrado en el Usuario DCU del portal web de leyendas riobambeñas, su contenido hace referencia a

\footnotetext{
${ }^{1}$ Escuela Superior Politécnica de Chimborazo, Facultad de Informática y Electrónica. Riobamba, Ecuador. mayra.pomaquero@espoch.edu.ec

${ }^{2}$ Escuela Superior Politécnica de Chimborazo, Facultad de Informática y Electrónica. Riobamba, Ecuador. bertha.paredes@espoch.edu.ec

${ }^{3}$ Universidad Nacional de Chimborazo. Riobamba, Ecuador. andrea.hidrobo@unach.edu.ec

${ }^{4}$ Escuela Superior Politécnica de Chimborazo, Facultad de Informática y Electrónica. Riobamba, Ecuador. f_calderon@espoch.edu.ec
} 
dimensiones culturales, trabajadas a partir de técnicas de diseño y cuyo resultado se ve reflejado en plataformas digitales. Para evaluar los diseños realizados, se ha llevado a cabo dos test de usuarios basados en los principios heurísticos y aplicados a dos grupos de usuarios entre 18 y 26 años de la Escuela superior Politécnica de Chimborazo. Los resultados están relacionados principalmente con la experiencia proporcionada por el nuevo diseño, que ha sido valorado muy positivamente por los estudiantes, y se obtiene como resultado que la creación de portales web pensados en satisfacer las necesidades del usuario y la aplicación de test previo a la publicación de los portales permite tener sitios web atractivos y que satisfagan los criterios de una web efectiva.

Palabras claves: Diseño web, user design, difusión cultural, leyendas riobambeñas

\section{Introducción.}

La presente investigación deriva de la relación entre la calidad y la utilidad de los contenidos, publicados en portales web. La aplicación del caso vincula estrechamente la dimensión cultural entendida genéricamente como un sistema de valores y creencias convertidas en formas de constitución de una sociedad. Estas dimensiones culturales son entendidas bajo metodologías y procedimientos de (DCU), el diseño centrado en el usuario para, (Galeano, 2017) y como primer acercamiento al concepto es; conocer las particularidades de los usuarios para reflejarlas en la interacción y apariencia que ofrece la interfaz y de esta manera aumentar su efectividad y calidad. El DCU como filosofía sitúa al usuario en el centro del proceso de diseño, y que busca garantizar el éxito de la aplicación o producto bajo esta premisa. Muriel Garreta y Enric Mor describe al DCU como una "metodología de desarrollo: una forma de planificar los proyectos y un conjunto de métodos que se pueden utilizar en cada una de las principales fases".

Esta filosofía es aplicable a todos los productos pero aquellos ligados a la tecnología son los que han trabajado sobre este camino para conseguir resultados deseados. De acuerdo a la norma ISO 13407, podemos desgranar este proceso en cuatro fases :

Entender y especificar el contexto de uso: Identificar a los usuarios y sus condiciones.

- Especificar requisitos: Identificar los objetivos del usuario y del proveedor .

- Producir soluciones de diseño: Desde las primeras soluciones conceptuales hasta la solución final de diseño.

- Evaluación: Es la fase más importante del proceso, en la que se validan las soluciones de diseño (el sistema satisface los requisitos) o por el contrario se detectan problemas de usabilidad, normalmente a través de test con usuarios. Al respecto de la usabilidad Cancio \& Bergues (2013) señalan que "el término ha sido utilizado para analizar aquellos factores que contribuyen a que un sitio web resulte fácil de utilizar", a esta idea general se le añade que Hassan, Fernández, \& Iazza, (2004) describen a este paso como el más importante dentro del proceso. 
Figura 1. Proceso DCU

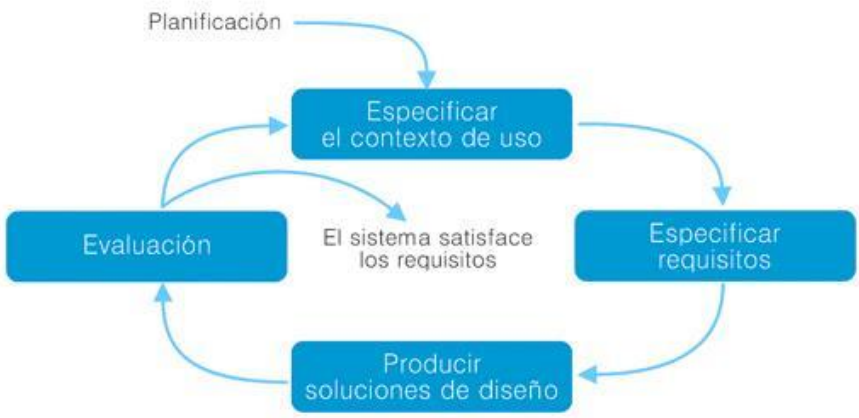

Fuente: nosolousabilidad.com

El diseño de experiencia deque usuario es un conjunto de métodos aplicados al proceso de experiencias (Allanwood \& Beare, 2015) y es así que los diseñadores se centran en crear experiencias interactivas de calidad . En UXD un diseño es eficaz cuando logra satisfacer todos los requerimientos y necesidades del usuario para la que ha sido creada. (Steve Krug, 2006) afirma que para el usuario el diseño debe ser "obvio, claro, evidente y fácil de entender".

Finalmente, el aspecto cultural de la investigación considera a la misma desde tres perspectivas. La primera; la cultura como un procesos colectivo de creación y recreación, la segunda; la cultura como herencia acumulada de generaciones anteriores, y finalmente, la cultura como conjunto de elementos dinámicos que pueden ser transferidos de grupo a grupo $\mathrm{y}$ en su caso aceptados, reinterpretados o rechazados por grupos sociales diversos.(Stavenhagen, 1986)

Estas perspectivas de cultura evidencian el estudio antropológico de las leyendas riobambeñas que se encuentran recogidas en el inventario de las leyendas del cantón Riobamba (Idrobo C., Pomaquero , Calderón \& Hidrobo , 2018) y tiene como objetivo difundir las principales leyendas del cantón y fortalecer la identidad de la población riobambeña, que según la experiencia analizada por Harris y Harris (2011) deriva que el uso de TICS logra fortalecer la identidad cultural y reduce la brecha digital de los pueblos de Malasia en dónde re realizó la experiencia, (López-Vicent, P., Sánchez Vera, M., \& Solano Fernández, I. 2013).

Con estos antecedentes, este artículo tiene como objetivo realizar un aporte al mejoramiento de la creación de sitios web enfocados en difundir la cultura , utilizar el diseño centrado en el usuario y puntualmente la usabilidad como conceptos que permitan a los usuarios obtener 
información de calidad al mismo tiempo que afianzan su identidad a través del análisis del portal web desarrollado para difundir las leyendas de Riobamba.

Figura 2. Página inicial beta

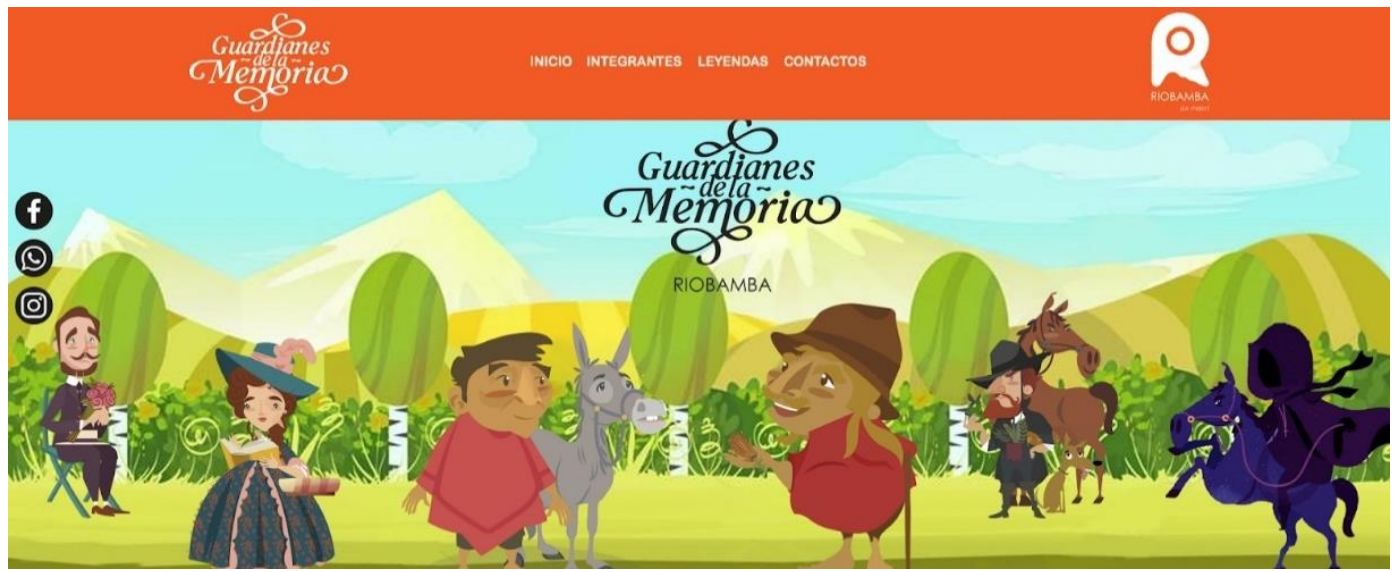

Fuente: Esteban Cajamarca

\section{Metodologia.}

Existen diferentes metodologías para la evaluación de un sitio web, para este estudio se utilizó la evaluación heurística a través de los 10 principios planteados por Jakob Nielsen (1995) que permiten medir la calidad de la página web. Estos principios son:

- Visibilidad del estado del sistema, El usuario siempre debe estar informado de lo que pasa en el sitio.

- Relación entre el sistema y el mundo real, Es sistema debe tener un lenguaje familiar para el usuario.

- Control y libertad del usuario, Dar al usuario la posibilidad de corregir el error.

- Consistencia y estándares, seguir las convenciones establecidas para ciertos iconos, usar los mismos al momento de estar en la versión responsive y no inventar nuevos.

- Prevención de errores, se trata de evitar cualquier error que el usuario pueda cometer.

- Reconocer antes que recordar, se debe ayudar al usuario a no memorizar.

- Flexibilidad y eficiencia de uso, se trata de conseguir que cualquiera pueda navegar por la web.

- Diseño estético y minimalista, la página web deben tener la información necesaria para no distraer al usuario con cosas molestas.

- Ayudar a los usuarios a reconocer, diagnosticar y corregir los errores. 
- Ayuda y documentación, basándose en estos principios se intenta que el usuario no tenga que usar documentos de ayuda para poder navegar dentro de un sitio web, pero siempre es necesario tener un manual sencillo de funcionamiento.

Vinculado al concepto anterior, Nielsen (2000), argumenta que aplicar un test a 5 usuarios permite revelar alrededor del $85 \%$ de los problemas de usabilidad del sitio o software. Referente a los test Gilbert Cockton (2003) menciona que solo una pequeña muestra de problemas pueden ser hallados bajo este método y que muchos otros pueden no ser hallados, aún si éstos son muy relevantes para el proyecto. Laura Faulkner (2003) indica que al realizar un test con 10 personas, el promedio de hallazgos es de $95 \%$ con un mínimo de $82 \%$, lo que sugiere una notoria mejora respecto al estudio con 5 usuarios.

En base a estas afirmaciones, se aplicó un primer test en la versión beta del portal a 10 usuarios en un rango de edad comprendido entre 18 y 26 años. Cada usuario en una computadora navegó por 15 minutos en el sitio web y posterior a eso respondió al test de preguntas cerradas y calificaron a la aplicación entre 0 y 10 puntos. Una vez analizadas las respuestas del primer test se procedió a realizar las mejoras en las áreas débiles de la página web y aplicar el misma prueba a un segundo grupo con las mismas características.

Figura 3. Gráfico de Nielsen

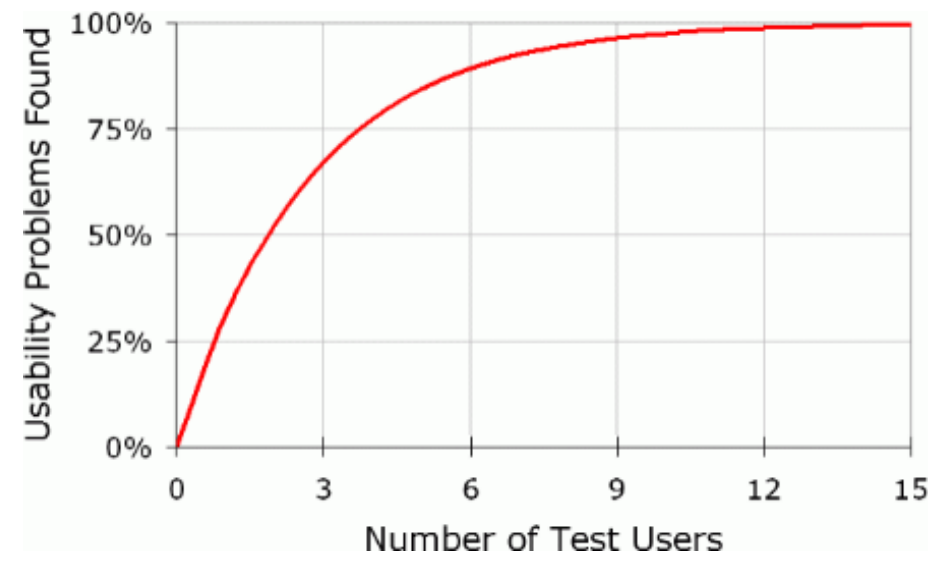

Fuente: blog.ida.co

\section{Resultados.}

La tabla 1 muestra las preguntas y los resultados obtenidos en el primer test, en dónde a través de 12 preguntas se evaluó los principios de Nielsen, todas las preguntas fueron cerradas con la opción de $\mathrm{Si}$ y No, la selección de la respuesta positiva determina la efectividad del parámetro, a excepción de la pregunta 10 que si se responde negativamente cumple efectivamente el parámetro. 
De la misma manera la tabla 2 refleja los resultados obtenidos en el test realizado luego de analizar los primeros datos y mejorar el portal web.

\section{Tabla 1. Respuestas grupo 1 primer test}

\begin{tabular}{|c|c|c|c|}
\hline N.- & Pregunta & SI & NO \\
\hline 1 & $\begin{array}{l}\text { ¿Cada pantalla de la interfaz contiene un título o } \\
\text { encabezado que la describe? }\end{array}$ & 1 & 9 \\
\hline 2 & $\begin{array}{l}\text { ¿El diseño de los íconos y su estética es equilibrada en todo } \\
\text { el sistema? }\end{array}$ & 8 & 2 \\
\hline 3 & $\begin{array}{l}\text { ¿Los nombres de los menús y textos son fáciles de } \\
\text { entender? }\end{array}$ & 7 & 3 \\
\hline 4 & ¿Encontró la sección de ayuda? & 4 & 6 \\
\hline 5 & $\begin{array}{l}\text { ¿Es posible cancelar alguna operación que se encuentre en } \\
\text { proceso? }\end{array}$ & 6 & 4 \\
\hline 6 & ¿Existen opciones de editar dentro del sistema? & 0 & 10 \\
\hline 7 & ¿Las opciones del menú son lógicas y distinguibles? & 8 & 2 \\
\hline 8 & ¿La navegación entre pantallas es simple y visible? & 10 & 0 \\
\hline 9 & $\begin{array}{l}\text { ¿El espacio en blanco es usado para crear simetría y guiar al } \\
\text { usuario para una navegación adecuada? }\end{array}$ & 8 & 2 \\
\hline 10 & ¿El formulario de registro presentó alguna dificultad? & 7 & 3 \\
\hline 11 & $\begin{array}{l}\text { ¿Existe algún elemento que ayudo a entender rápidamente } \\
\text { de que se trata el sitio web? }\end{array}$ & 9 & 1 \\
\hline 12 & ¿La información es fácil de recordar? & 8 & 2 \\
\hline
\end{tabular}

Fuente: test de usuario

Elaborado por: Los autores

Tabla 2. Respuestas grupo 2 segundo test

\begin{tabular}{llll}
\hline $\mathbf{N} .-$ & Pregunta & SI & NO \\
\hline $\mathbf{1}$ & $\begin{array}{l}\text { ¿Cada pantalla de la interfaz contiene un título o } \\
\text { encabezado que la describe? }\end{array}$ & 8 & 2 \\
$\mathbf{2}$ & $\begin{array}{l}\text { ¿El diseño de los íconos y su estética es equilibrada en } \\
\text { todo el sistema? }\end{array}$ & 7 & 3 \\
$\mathbf{3}$ & $\begin{array}{l}\text { ¿Los nombres de los menús y textos son fáciles de } \\
\text { entender? }\end{array}$ & 8 & 2 \\
$\mathbf{4}$ & $\begin{array}{l}\text { ¿Encontró la sección de ayuda? } \\
\mathbf{5}\end{array} \quad \begin{array}{l}\text { ¿Es posible cancelar alguna operación que se } \\
\text { encuentre en proceso? }\end{array}$ & 8 & 2
\end{tabular}




\begin{tabular}{|c|c|c|}
\hline 6 & ¿Existen opciones de editar dentro del sistema? & 6 \\
\hline 7 & ¿Las opciones del menú son lógicas y distinguibles? & 8 \\
\hline 8 & ¿La navegación entre pantallas es simple y visible? & $\begin{array}{l}1 \\
0\end{array}$ \\
\hline 9 & $\begin{array}{l}\text { ¿El espacio en blanco es usado para crear simetría y } \\
\text { guiar al usuario para una navegación adecuada? }\end{array}$ & 7 \\
\hline 10 & ¿El formulario de registro presentó alguna dificultad? & 4 \\
\hline 11 & $\begin{array}{l}\text { ¿Existe algún elemento que ayudo a entender } \\
\text { rápidamente de que se trata el sitio web? }\end{array}$ & 9 \\
\hline 12 & ¿La información es fácil de recordar? & 7 \\
\hline
\end{tabular}

Fuente: test de usuario

Elaborado por: Los autores

La tabla 3 muestra una comparativa entre los resultados del primer y segundo test, que fue aplicado a grupos distintos de usuarios y dónde se ven las variaciones de los resultados en ambos test.

Tabla 3. Comparativa resultados test

\begin{tabular}{ccccc}
\hline Pregunta & T1 & T2 & T1 & T2 \\
& SI & SI & NO & NO \\
$\mathbf{1}$ & 1 & 8 & 9 & 2 \\
$\mathbf{2}$ & 8 & 7 & 2 & 3 \\
$\mathbf{3}$ & 7 & 8 & 3 & 2 \\
$\mathbf{4}$ & 4 & 7 & 6 & 3 \\
$\mathbf{5}$ & 6 & 8 & 4 & 2 \\
$\mathbf{6}$ & 0 & 6 & 10 & 4 \\
$\mathbf{7}$ & 8 & 8 & 2 & 2 \\
$\mathbf{8}$ & 10 & 10 & 0 & 0 \\
$\mathbf{9}$ & 8 & 7 & 2 & 2 \\
$\mathbf{1 0}$ & $\mathbf{7}$ & $\mathbf{4}$ & $\mathbf{3}$ & $\mathbf{6}$ \\
$\mathbf{1 1}$ & 9 & 9 & 1 & 1 \\
$\mathbf{1 2}$ & 8 & 7 & 2 & 3 \\
\end{tabular}

Fuente: test de usuario

Elaborado por: Los autores

La tabla 4 muestra la calificación obtenida del portal web, en un rango de 0 a 10 puntos los usuarios dieron una nota al portal y se observa que en el primer test el promedio fue de 7.1, luego de mejorar los puntos débiles los usuarios en promedio puntuaron a la web con 8.1, que aunque mejora aún no cumple con satisfacción los parámetros de Nielsen. 
Tabla 4. Calificación de los usuarios

\begin{tabular}{ccc}
\hline Usuario & T1 & T2 \\
\hline $\mathbf{1}$ & 8 & 7 \\
$\mathbf{2}$ & 7 & 9 \\
$\mathbf{3}$ & 7 & 9 \\
$\mathbf{4}$ & 6 & 8 \\
$\mathbf{5}$ & 7 & 7 \\
$\mathbf{6}$ & 6 & 8 \\
$\mathbf{7}$ & 6 & 8 \\
$\mathbf{8}$ & 9 & 10 \\
$\mathbf{9}$ & 8 & 7 \\
10 & 7 & 8 \\
Promedio/10 & 7.1 & 8.1 \\
\hline
\end{tabular}

Fuente: test de usuario

Elaborado por: Los autores

\section{Discusión.}

Se inicia básicamente con la importancia de la información y difusión cultural en determinados portales web, para esto (Magán, 2016) menciona que la leyenda además de contar con una forma expresiva, también es una forma de comunicación, y es ahí donde radica la importancia de su uso en el internet y en la creación de portales web, donde se vinculen estos valiosos contenidos culturales con plataformas digitales que permitan además de su difusión su permanencia.

Esta información cultural expresada en forma de leyendas, cuentan con la metodología de Diseño basado en el usuario DCU donde los principios de Nielsen determinan entre otras cosas soluciones visuales y funcionales a diversos requerimientos comunicativos, que para (Galeano, 2017) son soluciones que en su conjunto procuran brindarle al usuario final una buena "experiencia de uso". Así en la investigación son evaluados aspectos que engloban las mencionadas soluciones como: el diseño, la navegación, pertinencia etc. Todos estos conjugados armonicamente a travez del interfaz, (Hartson, 1998) menciona que "la interfaz es la aplicación" puesto que es la parte que ven y a través de la cual interactúan los usuarios.

Finalmente los elementos evaluados no solo incluyen elementos formales como el interfaz sino, también la arquitectura, la estructura y la organización, en otras palabras, el componente no visible del diseño, que para la investigación presentan resultados favorables. 
En conclusión el diseño centrado en el usuario como proceso de creación de un portal web, y la aplicabilidad de los test basados en los principios de Nielsen, han permitido que el portal web desarrollado maneje los parámetros idóneos para su publicación, se verifica que los resultados son confiables debido a las similitudes en las respuestas obtenidas en las dos pruebas, y el mejoramiento de las mismas a partir de identificar los aspectos técnicos que se necesitaban cambiar.

\section{Conclusiones.}

- El diseño centrado en el usuario constituye una estrategia de desarrollo en el ámbito cultural, cuya información contribuye a la conservación de manifestaciones culturales de la región.

- La aplicación de los test de usuario en etapas temprana del diseño y desarrollo permiten identificar claramente los puntos débiles del producto web y de esta manera mejorarlo previo a la publicación final del proyecto, lo cual aplicado a todo tipo de sitios constituye un ahorro importante de recursos.

- La evaluación heurística y la aplicación de los principios de Nielsen reúne los puntos clave del diseño web, y aunque recoge de manera adecuada los criterios es importante que además de esta evaluación si se cuenta con los recursos se pueda utilizar otros instrumentos y medios tales como entrevistas, diseño de perfiles de usuario y grupos focales para la validación de los portales web.

\section{Referencias bibliográficas.}

Allanwood, G., \& Beare, P. (2015). Diseño de experiencias de usuario: cómo crear diseños que gustan realmente a los usuarios. Parramón Paidotribo.

Bevan, N., Barnum, C., Cockton, G., Nielsen, J., Spool, J., \& Wixon, D. (2003, April). The magic number 5: is it enough for web testing?. In CHI'03 extended abstracts on Human factors in computing systems (pp. 698-699). ACM.

Cancio, L. P., \& Bergues, M. M. (2013). Usabilidad de los sitios Web, los métodos y las técnicas para la evaluación. Revista Cubana de Información en Ciencias de la Salud (ACIMED), 24(2), 176-194.

Faulkner, L. (2003). Beyond the five-user assumption: Benefits of increased sample sizes in usability testing. Behavior Research Methods, Instruments, \& Computers, 35(3), 379-383. 
Galeano, R. (2017). Diseño centrado en el usuario. Revista q, 2(4).

Hartson, H.R. (1998). Human-computer interaction: Interdisciplinary roots and trends. En: Journal of Systems and Software, Noviembre 1998, v. 43, n. 2, pp. 103-118.

Hassan, Y., Martín Fernández, F. J., \& Iazza, G. (2004). Diseño web centrado en el usuario: usabilidad y arquitectura de la información. Hipertext. net, (2).

Idrobo, C., Pomaquero, Y., Calderón Cruz, F. A., Nina, H., \& Fernanda, A. (2018). Salvaguarda de las leyendas del cantón Riobamba como patrimonio intangible. Caribeña de Ciencias Sociales, (junio).

Krug, S. (2006). No me hagas pensar: una aproximación a la usabilidad en la web (No. Gi2974). PEARSON.

López-Vicent, P., Sánchez Vera, M., \& Solano Fernández, I. (2013). Las TIC para el desarrollo de la identidad digital y cultural de pueblos originarios. Papeles de Trabajo sobre Cultura, Educación y Desarrollo Humano, 10(1), 1-18.

Magán, P. M. (2016). Las leyendas y su valor didáctico. Centro Virtual Cervantes, 400, 392-403.

Mor, E., Domingo, M. G., \& Galofré, M. (2007, September). Diseño Centrado en el Usuario en Entornos Virtuales de Aprendizaje, de la Usabilidad a la Experiencia del Estudiante. In SPDECE.

Nielsen, J. (1995). How to conduct a heuristic evaluation. Nielsen Norman Group, 1, 1-8.

Nielsen, J. (2000). Usabilidad. Diseño de páginas Web. DE INFORMACIÓN

Nielsen, J., \& Molich, R. (1990, March). Heuristic evaluation of user interfaces. In Proceedings of the SIGCHI conference on Human factors in computing systems (pp. 249-256). ACM.

Stavenhagen, R. (1986). cultura popular y creación intelectual.pdf.

Rodríguez, S., \& NSaaverda, C.. (2018). Las TIC: Una oportunidad para fortalecer procesos de enseñanza en el marco del modelo escuela nueva. ESPACIOS . Revista electrónica http://www.revistaespacios.com/a18v39n49/a18v39n49p33.pdf 


\section{PARA CITAR EL ARTÍCULO INDEXADO.}

Pomaquero Yuquilema, M., Paredes Calderón, B., Hidrobo Nina, A., \& Calderón Cruz, F. (2019). Diseño centrado en el usuario: Usabilidad web del portal de difusión cultural de leyendas $\begin{array}{llll}\text { riobambeñas. } & \text { Ciencia } & \text { 3igital, } & \text { 133-143. }\end{array}$

https://doi.org/10.33262/cienciadigital.v3i3.2.1.787

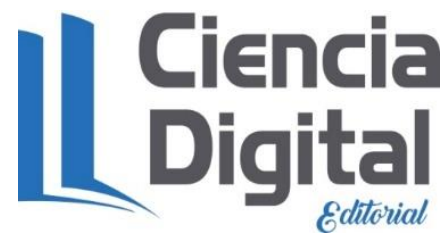

El artículo que se publica es de exclusiva responsabilidad de los autores y no necesariamente reflejan el pensamiento de la Revista Ciencia Digital.

El artículo queda en propiedad de la revista y, por tanto, su publicación parcial y/o total en otro medio tiene que ser autorizado por el director de la Revista Ciencia Digital.
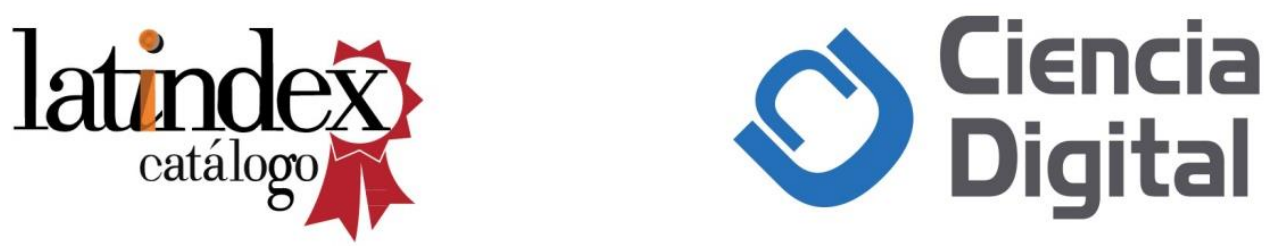